\title{
SQUID-detected FMR: Resonance in single crystalline and polycrystalline yttrium iron garnet
}

J. M. O'Reilly, and P. Stamenov

Citation: Review of Scientific Instruments 89, 044701 (2018); doi: 10.1063/1.5009731

View online: https://doi.org/10.1063/1.5009731

View Table of Contents: http://aip.scitation.org/toc/rsi/89/4

Published by the American Institute of Physics

\section{Articles you may be interested in}

Optically coupled methods for microwave impedance microscopy

Review of Scientific Instruments 89, 043703 (2018); 10.1063/1.5011391

High-resolution photoluminescence electro-modulation microscopy by scanning lock-in

Review of Scientific Instruments 89, 043705 (2018); 10.1063/1.5010281

Direct detection of spin Nernst effect in platinum

Applied Physics Letters 112, 162401 (2018); 10.1063/1.5021731

Vector network analyzer ferromagnetic resonance spectrometer with field differential detection

Review of Scientific Instruments 89, 053901 (2018); 10.1063/1.5022762

In situ/non-contact superfluid density measurement apparatus

Review of Scientific Instruments 89, 043901 (2018); 10.1063/1.5012043

Note: Commercial SQUID magnetometer-compatible NMR probe and its application for studying a quantum magnet

Review of Scientific Instruments 89, 046101 (2018); 10.1063/1.5023675

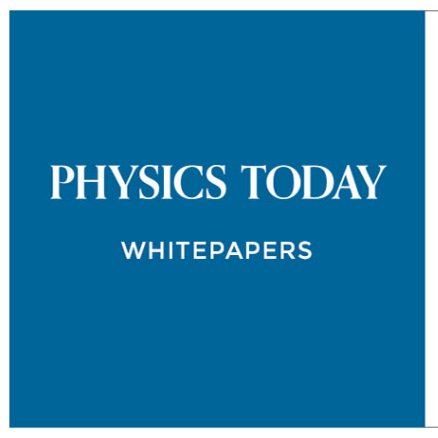

MANACER'S GUIDE

Accelerate R\&D with

Multiphysics Simulation
READ NOW

PRESENTED BY

$\checkmark \subset \bigcirc M S \bigcirc L$ 


\title{
SQUID-detected FMR: Resonance in single crystalline and polycrystalline yttrium iron garnet
}

\author{
J. M. O'Reillya) and P. Stamenov' \\ School of Physics, Centre for Research on Adaptive Nanostructures and Nanodevices (CRANN) and Advanced \\ Materials Bio-Engineering Research Centre (AMBER), Trinity College Dublin, Dublin 2, Ireland
}

(Received 19 October 2017; accepted 20 March 2018; published online 9 April 2018)

\begin{abstract}
Here two new techniques for the detection of broadband $(100 \mathrm{MHz}-20 \mathrm{GHz})$ ferromagnetic resonance (FMR)/ferrimagnetic resonance in single and poly-crystalline materials, which rely on SQUID-based gradiometry detection of small changes in the magnetisation, are developed. In the first method, small changes in the along-the-applied-field projection of the coupled magnetic moment $\left(\Delta m_{z}\right)$ are detected as the material is driven into resonance. Absolute measurement of the longitudinal component of the magnetisation and the resonance induced lowering of this moment makes estimation of the precession cone angle accessible, which is typically difficult to extract using conventional cavity or stripline based detection methods. The second method invokes the change in $\Delta m_{z}$ with the resonance-induced thermal heating $\left(\frac{\mathrm{d} m_{z}}{\mathrm{~d} T}\right)$. Magnetisation dynamics in bulk $\mathrm{Y}_{3} \mathrm{Fe}_{5} \mathrm{O}_{12}$ are observed over a broad range of experimental temperatures $(4 \mathrm{~K}-400 \mathrm{~K})$ and fields $(10-500 \mathrm{mT})$. The inhomogeneous microwave excitation allows for the observation of higher magnetostatic modes and the convenient tracking of very broad resonances. The two SQUID-detection techniques when combined with conventional broadband vector network analyser-FMR, low-frequency magnetic susceptibility, and DC magnetometry, all easily realised, essentially concurrently, using the same module, greatly expand the amount of static and dynamic information accessible. Published by AIP Publishing. https://doi.org/10.1063/1.5009731
\end{abstract}

\section{INTRODUCTION}

Resonance detection methods have been used extensively to examine the magnetisation dynamics in ferromagnetic and ferrimagnetic systems since the effect's discovery in the late 1940s. ${ }^{1,2}$ The most commonly used methods for characterising ferromagnetic resonance (FMR) rely on either resonant cavity or microstrip-based inductive detection of the reflected signals that originate from subjecting the material to an external microwave field and inferring the resonant absorption by comparing the incident and reflected signals. These standard techniques for the measurement of FMR do not provide for the direct and absolute extraction of the precession cone angle. Quantitative information for the strength of the resonance is rather difficult to obtain. Recently there has been a renewed interest in high-frequency FMR and materials suitable for operation in the bands beyond $10 \mathrm{GHz}$. As such, non-inductive techniques have emerged in the field, most notably FMR force microscopy (FMRFM), 3,4 X-ray magnetic circular dichroism (XMCD) detection, ${ }^{5}$ and optically based methods, such as magneto-optic Kerr effect (MOKE). ${ }^{6,7}$

In this paper, a method of detecting FMR by directly measuring the change in the longitudinal component of the magnetisation $\Delta m_{z}$ when a material is in resonance, using SQUID magnetometry, is demonstrated. Such measurements have only been made previously using MOKE-based techniques $^{8}$ for FMR detection. While longitudinal SQUID-based

a)Electronic mail: oreillj5@tcd.ie

b)Electronic mail: stamenop@tcd.ie detection has been applied before to the NMR case, ${ }^{9}$ there are specific differences associated primarily with the much higher resonance frequencies, much shorter decay times, and much stronger coupling to the measurement system in the case of FMR. Here the FM material is considered as a macro-spin system, as in Fig. 1, where the length of the magnetisation vector, which is nominally saturated along the $z$ direction $m_{z}$, remains constant. As the system is excited at its resonance frequency, it will absorb microwave excitation power and the precession cone will open. Opening of the precession cone leads to lowering of the $m_{z}$ projection of the magnetic moment, which is approximately quadratic as a function of the cone opening as inferred from the cosine expansion at small angles. Using a widely available commercial SQUID magnetometer (Quantum Design magnetic property measurement system MPMS $^{\circledR} 5 \mathrm{XL}^{10}$ ), we can simultaneously obtain absolute measures of $m_{z}, \Delta m_{z}$, and the absorbed power and can subsequently gain a quantitative insight into the precession cone angle of the sample (material studied) and its dependence on the amount of resonantly absorbed power.

Supplementary to this, we demonstrate magnetic resonance detection by monitoring $\Delta m_{z}$ as a function of changing temperature caused by absorption of microwave power (heating), using SQUID magnetometry. Here, the strong interaction with a modulated high-frequency signal which leads to precession cone opening has the added effect of periodic heating, which is especially pronounced at resonance. This oscillatory heating induces changes in $m_{z}$, which are directly observed in the SQUID output signal. This thermal detection, while not inherently absolute, exhibits excellent sensitivity and resolution, especially at low temperatures. 

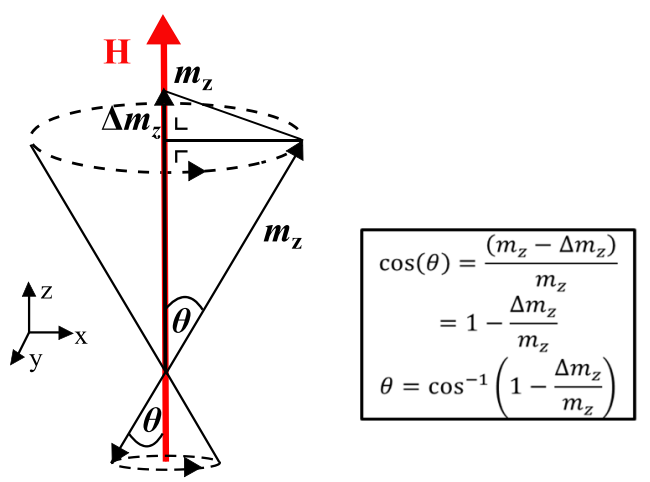

FIG. 1. Change in projection of the magnetic moment along $z\left(\Delta m_{z}\right)$ during resonance.

This work will discuss the application of the aforementioned techniques in analysing the magnetisation dynamics in probably the most prototypical ferrimagnetic insulator, yttrium-iron garnet $\left(\mathrm{Y}_{3} \mathrm{Fe}_{5} \mathrm{O}_{12}-\mathrm{YIG}\right)$. YIG, due to its unique magnetic and thermal properties: very narrow resonancelinewidth, low spin-damping at microwave frequencies, and high $T_{c} \approx 560 \mathrm{~K}$, has been the basis of most insulatorbased radio frequency and microwave devices and the standard test material for novel resonance phenomena in spintronics (i.e., spin pumping). ${ }^{11-15}$ YIG has a complex crystal structure with 80 atoms per unit cell, consisting of four formula units of $\mathrm{Y}_{3} \mathrm{Fe}_{2}{ }^{3+} \mathrm{Fe}_{3}{ }^{3+} \mathrm{O}_{12}{ }^{2-}$ per unit cell. The $\mathrm{Fe}$ atoms occupy two crystallographic positions, 16 a-sites octahedrally coordinated by oxygen, and $24 \mathrm{~d}$-sites tetrahedrally coordinated by oxygen, with an unbalanced, trivalent $\mathrm{Fe}$ atom giving rise to a net spontaneous magnetisation. ${ }^{16}$ In the microwave regime, YIG is typically described using a two-sublattice approximation and the resonant energy absorption by the macroscopic magnetisation is then interpreted using Kittel's theory for ferromagnetic resonance. ${ }^{17}$ Despite its profuse industrial applications, much is still to be understood about the details of magnetisation dynamics at low temperatures as YIG's magnonic spectra are shown to have 20 distinct magnon branches, which are not all necessarily in phase with each other. ${ }^{18-21}$ This study characterizes the gyroscopic properties of both bulk poly- and single-crystalline yttrium iron garnet, as an illustration of the capabilities of the newly developed SQUID-FMR (SFMR).

\section{EXPERIMENTAL}

Figure 2 shows the design of the resonator consisting of a stainless steel coaxial cable for connecting to the swept frequency source and the FR4 (woven fibreglass and epoxy resin)-based microstrip transmission line shorted with a $50 \Omega$ load resistance $(0.5 \mathrm{~mm}$ in size $)$, encased in a thin walled copper shielding. The adjusted $50 \Omega$ termination minimizes the amplitude of the back-reflected wave at essentially all frequencies used, leaving only a small amplitude standing wave, the nodes of which vary in spatial position as a function of frequency. The sample can be positioned in the central (40 mm-long) region of the microstrip. Since the sample position is typically fixed during measurements, the small amplitude standing waves would project maximal $B$-field intensity

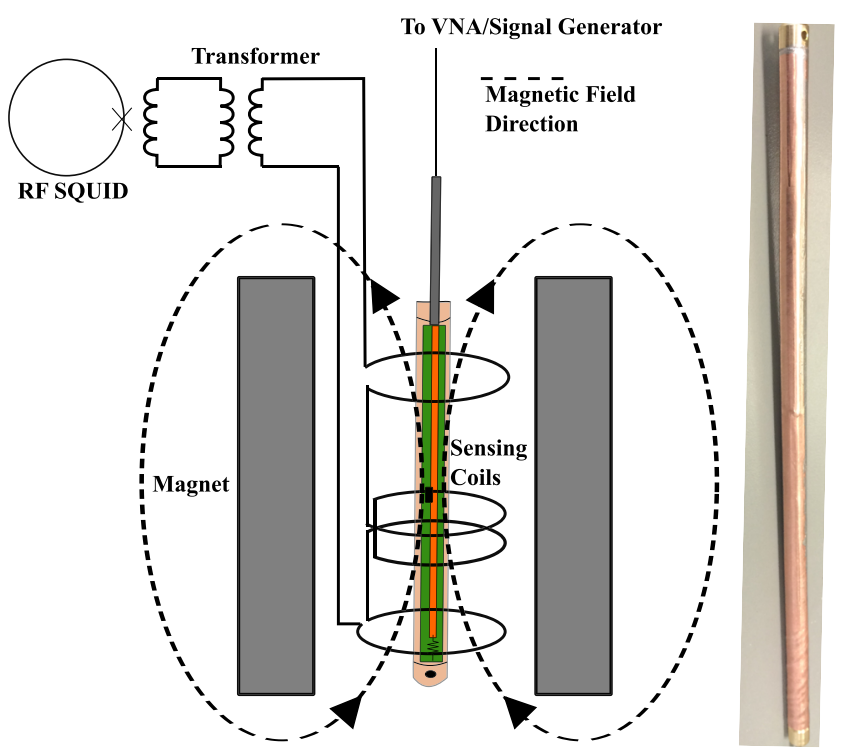

FIG. 2. Schematic of the SQUID-based FMR detector, showing the FR4based microstrip contained within copper shielding, at the centre of the SQUID magnetometer sample space.

only at a certain comb of frequencies. The $z$-axis profile of the $B$-field distribution above the microstrip is essentially cosine in nature, with its zeroth node located at the $50 \Omega$ terminator. The majority of microwave loss is incurred within the cryogenic coaxial line, with about $3 \mathrm{~dB}$ of loss at $1 \mathrm{GHz}$ and $30 \mathrm{~dB}$ of loss at $18 \mathrm{GHz}$. A further $0.5 \mathrm{~dB}$ of loss is incurred in the microstrip at $1 \mathrm{GHz}$, further increasing to $5 \mathrm{~dB}$ at $18 \mathrm{GHz}$. The overall loss, at $18 \mathrm{GHz}$, to the termination point is $35 \mathrm{~dB}$. This can be substantially improved upon by substituting the cryogenic coaxial line, with a low-loss alternative; however, this can only be done on expense of a substantial increment in the $z$-axis heat loss, particularly important for low-temperature measurements.

Two venting holes are positioned at the end of the shield, perpendicular to one another (the vertical one being blind). These serve the sole purpose of preventing implosion of the shielding during pump-down and have no particular microwave functionality as are located a couple of millimetres below the $50 \Omega$ termination of the microstrip. The $90^{\circ}$ angle creates a maximum in reflection, ensuring that stray microwave field from the microstrip will not interfere with the flux-locked SQUID loop. The rectangular prism of bulk, polycrystalline YIG (dimensions: $2.5 \times 1.6 \times 0.7 \mathrm{~mm}$ ) or polished single crystal YIG sphere $(D=300 \mu \mathrm{m})$, is mounted (using varnish) adjacent to the signal plane of the transmission line, where the external RF magnetic field is at a maximum. The sample assembly is placed in the centre of the sensing coils (four-turn $z$-axis gradiometer; see Fig. 2), which are inductively coupled to the RF SQUID within the cryogenic, vacuum environment of the superconducting magnet system.

The total $z$-axis magnetic moment projection is measured, as usual, by translating the sample vertically around the central position and processing the dependence of the coupled flux on distance. This component can be large and is processed using the lowest gain ranges of the SQUID amplifier. The moment typically is on the order of several $\mathrm{mAm}^{2}$. With 
the superconducting magnet in persistence mode and after a delay determined by a number of different physical factors (eddy current dissipation, on the scale of tens to hundreds of milliseconds, superconducting flux creep on the scale of seconds to minutes, to mention but a few), the superconducting transformer to the SQUID washer is quenched. The very large coupled flux is thereafter no longer measured. For the normal $M_{z}$ measurements, the SQUID is typically operated within much less than $1 \Phi_{0}$ of coupled flux (the response being flux-periodic, modulo $\Phi_{0}$ ). It is essentially impossible to measure changes in moment corresponding to $10^{-6}$ of $\Phi_{0}$, while using the flux-locked loop. Once the coupled flux is known within $1 \Phi_{0}$, a momentary quenching of the coupling transformer ensures that no super-current is flowing through the pick-up assembly and that only relative changes in flux (as a function of temperature, microwave absorption, etc.) are measured. Since the change in flux to change of sample moment ratio is well known (calibrated) for all gain ranges, the change in moment, relative to the total $M_{z}$, can be directly inferred. At this stage (while staying within $1 \Phi_{0}$ ), coupled fluxes of the order of $10^{-4} \Phi_{0}$ and below can be readily detected. It is at this point when the SQUID-FMR measurements begin. The above settling algorithm slows substantially down measurements of field dependences allowing for about one spectrum to be acquired every three to five minutes. Within this obligatory delay, other measurements can be readily performed, including DC magnetometry, low-frequency AC susceptibility, and recoil relaxometry. Measurements requiring the evolution of temperature at fixed field are much less restrictive and can often be performed "on the fly." A typical noise floor for such a detection system is below $3 \mathrm{nAm}^{2}$ with a time constant of the order of $1 \mathrm{~s}$. In measurement strategies where amplitude chopping or frequency modulation is utilised, the modulation frequency can be varied in the $1 \mathrm{mHz}$ to $20 \mathrm{kHz}$ range. This allows for at least two different measurement strategies, one athermal and one thermal as detailed later.

\section{RESULTS AND DISCUSSION}

The experimental setup may be operated as a conventional broadband (100 MHz-20 GHz) single-port vector network analyser (VNA)-FMR spectrum analyser, where FMR spectra can be obtained over a broad range of static fields and experimental temperatures. The unprocessed output signal appears as a complex series of peaks in the cumulative reflected amplitude, as shown in Fig. 3 for the polycrystalline sample, where the signal-to-noise ratio largely depends on the filtering and sampler used. In order to process the FMR signal, one must account for reflections due to discontinuities within the experimental apparatus. Modeling of the reflected signals' amplitude and phase is possible using transmission line theory, as seen in Figs. 3(a) and 3(b). However, modeling of all reflections within the experimental setup is not a trivial task due to the dispersive nature of the materials, FR4 and Polytetrafluoroethylene (PTFE) at high frequencies. If the setup were to be sufficiently compartmentalised, the reflections originating from each module could be calibrated (and de-embedded) and theoretically one could provide a more accurate model for all reflections, but this is beyond the scope of this work. At low frequencies, where

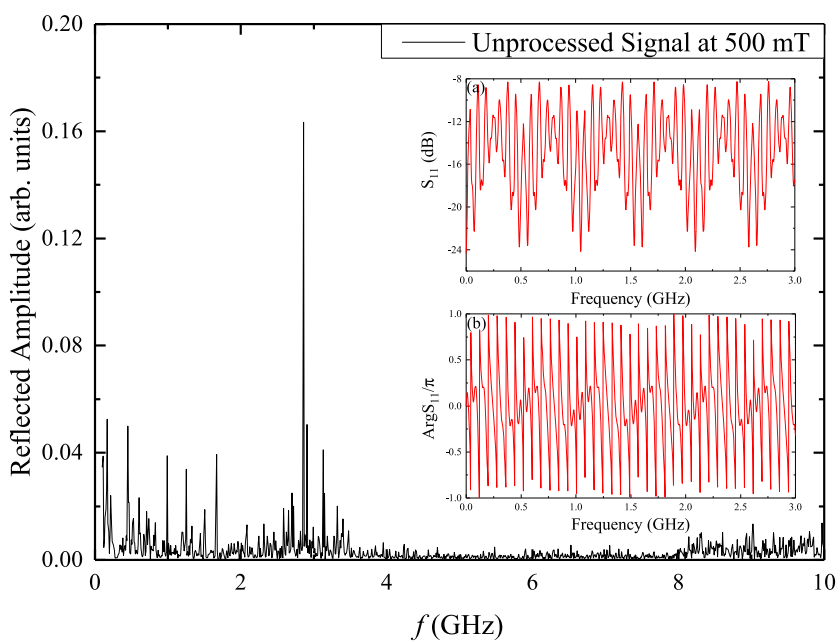

FIG. 3. Unprocessed VNA-FMR spectra in a $50 \mathrm{mT}$ static field. Insets display (a) the $S_{11}$ scattering parameters and (b) the phase as a function of frequency, simulated using transmission line theory.

good signal-to-noise ratios can be readily achieved, resonances can be simply tracked by subtracting a spectrum measured at remanence either in a multi-domain state or in complete saturation at fields large enough to place the resonance frequency of the lowest magnetostatic mode at frequencies way beyond the Nyquist threshold and the front end filter cutoff of the VNA. This process is illustrated in Fig. 4. The exacerbated broadening at very low fields is attributed to the large distribution of internal demagnetising fields in the specimen below magnetisation saturation. The line shape at fields below $25 \mathrm{mT}$ is not purely Lorentzian but rather a Gaussian convoluted Lorentzian, well fitted by a pseudo-Voigt function. The broadband (100 MHz-10 GHZ) FMR signals for the cuboid, shown in Fig. 5, are extracted from the unprocessed data by subtracting off-resonance background spectra and the fieldindependent peaks in the reflected amplitude, a process much more difficult at high frequencies. The resonant frequency $\left(f_{\text {res }}\right)$ is indicated by a sharp increase in the reflected amplitude which shifts to higher frequencies with increasing applied field, in agreement with Kittel's equation for ferromagnetic resonance. ${ }^{17}$ VNA-FMR spectra in Fig. 6 suggest the existence of two resonant modes which are strongly coupled to geometric resonances within the experimental apparatus. The higher

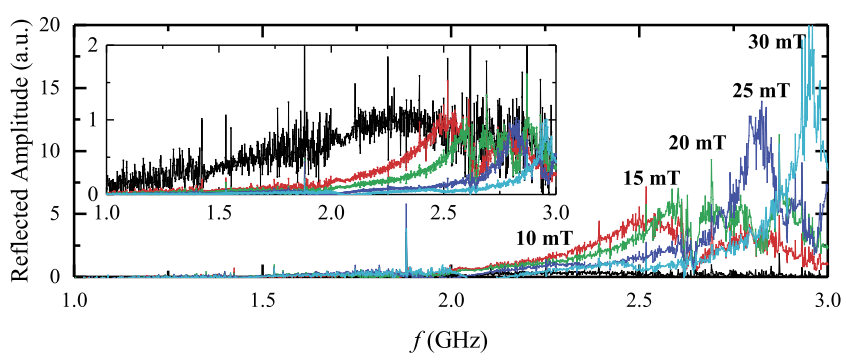

FIG. 4. Evolution of the resonant signal at low fields $(10-30 \mathrm{mT})$ for the polycrystalline cuboid. Inset shows the normalised amplitude for the same applied field values, in order to render the very broad, low-field resonance visible. The resonant signal for the $10 \mathrm{mT}$ applied field (black trace) is only rendered by normalisation. The sharp peaks at 1.8 and $2.4 \mathrm{GHz}$ are due to GSM and WiFi interference, respectively. 


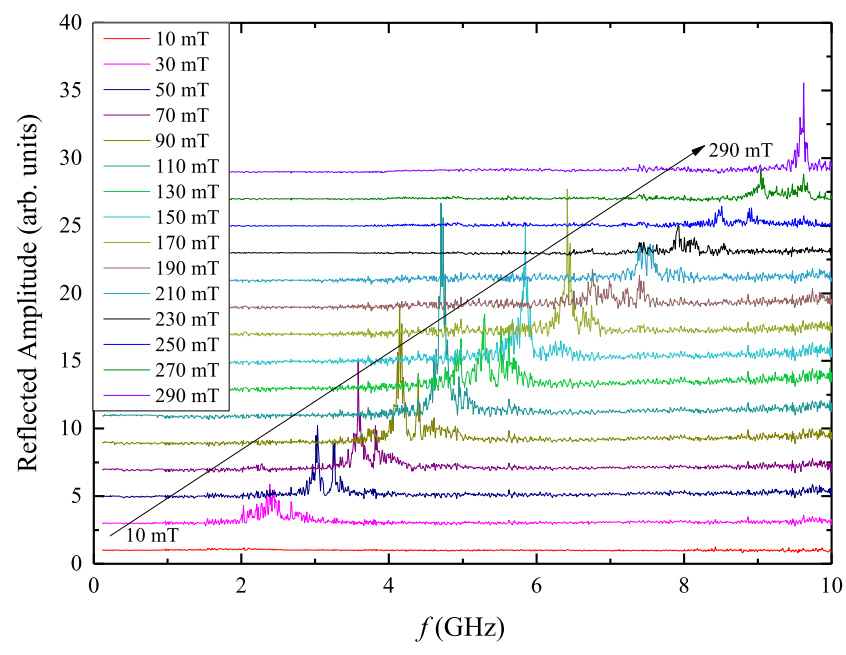

FIG. 5. VNA-FMR spectra for a rectangular prism of bulk YIG. Frequency dependence of the background subtracted reflected amplitude under a static field $\mu_{0 H}$.

and lower frequency modes are shown to have demagnetising fields of $110 \mathrm{mT}$ and $100 \mathrm{mT}$, respectively, and correspond to the two different demagnetizing directions $x$ and $y$. The gyromagnetic ratio $\gamma$ is measured as $27.8(3) \mathrm{GHz} / \mathrm{T}$.

Figure 7 shows tracing of the VNA-FMR resonance spectra with an applied magnetic field ranging from $70 \mathrm{mT}$ to $450 \mathrm{mT}$ for low temperature (a) $5 \mathrm{~K}$ and room temperature (b) $300 \mathrm{~K}$ for a $300 \mu \mathrm{m}$ diameter YIG sphere. Once again, enhancement, broadening, or splitting of the FMR spectra is observed at frequencies where there is strong coupling of the sphere's FMR mode with the electrostatic modes of the microstrip. The FMR spectra can be detected up to $14 \mathrm{GHz}$, beyond which the signals are greatly diminished, primarily due to the loss of microwave power reaching the sample, through the cryogenic coaxial line. A characteristic Lorentzian line shape (pseudo-Voigt shape parameter above 0.9 , out of unity) is observed for the range of applied fields. This is, unsurprisingly, much narrower than the one for the polycrystalline

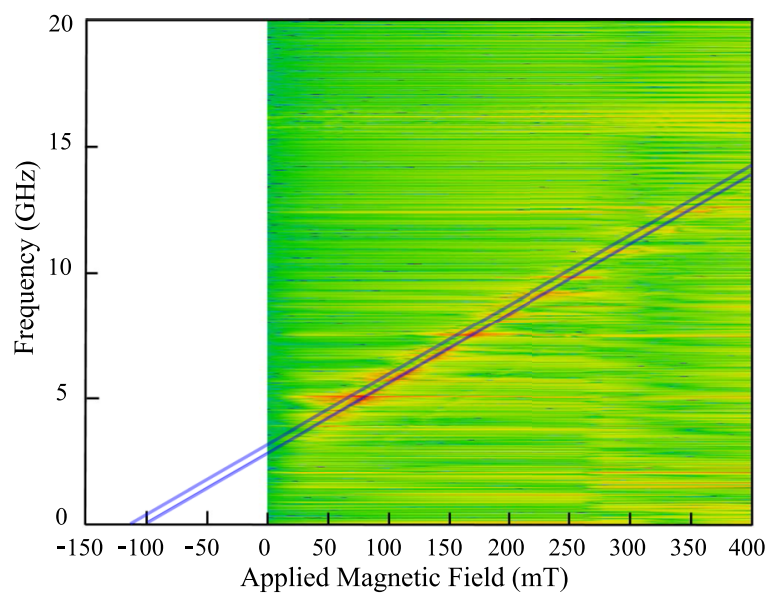

FIG. 6. VNA-FMR spectra of polycrystalline YIG obtained for $100 \mathrm{MHz}-$ $20 \mathrm{GHz}$ excitation frequency and 0-400 mT external applied field. Two resonant modes are identified which are strongly coupled to the transmission line electromagnetic modes. Demagnetising fields are determined; $H_{d 1}=100 \mathrm{mT}$, $H_{d 2}=110 \mathrm{mT}, \gamma=27.8(3) \mathrm{GHz} / \mathrm{T}$.

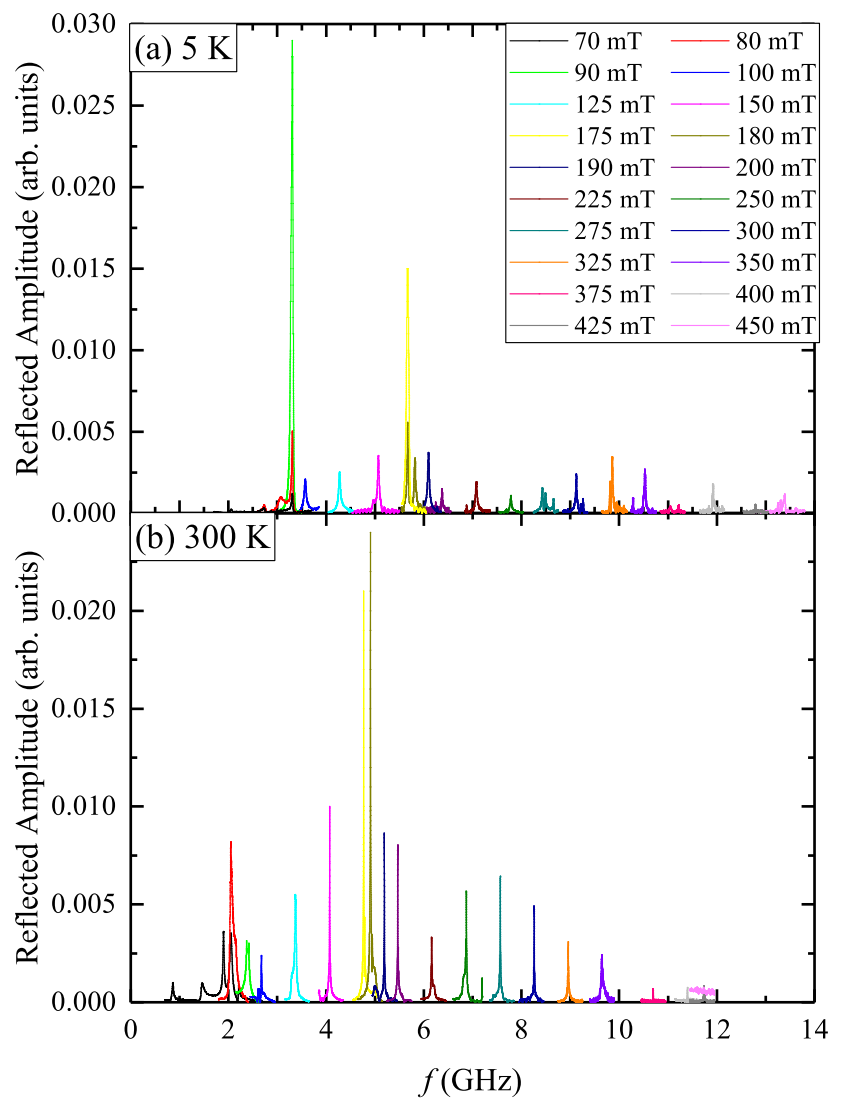

FIG. 7. VNA-FMR spectra for a single-crystal YIG sphere at (a) $5 \mathrm{~K}$ and (b) $300 \mathrm{~K}$. The observed linewidths are much narrower than those shown for the polycrystalline specimen (Fig. 5).

specimen, as in Fig. 5. Additional Gaussian inhomogeneity broadening is clearly present for the polycrystalline cuboid, as well established in the literature. ${ }^{22}$ No resonance could be observed for the single crystalline sphere at fields below $70 \mathrm{mT}$, which would be required for the analysis of the line shape below saturation and direct comparison of line shapes (in this regime) with polycrystals.

Figure 8 examines two resonant peaks for the YIG sphere obtained in a $150 \mathrm{mT}$ excitation field at $5 \mathrm{~K}$ and $300 \mathrm{~K}$. A shift in the resonance peak to a higher frequency is observed with decreasing temperature. Lateral broadening of the resonance peak is also observed. At $300 \mathrm{~K}$, the linewidth is measured as $8 \mathrm{MHz}$, which increases over fivefold to $44 \mathrm{MHz}$ at low temperatures. This temperature dependent linewidth broadening for polished spheres of single crystal YIG was first reported by Dillon in $1956 .^{23,24}$ This he attributed to the increase in the first order magnetocrystalline anisotropy constant $\frac{K_{1}}{M_{S}}$, with decreasing temperature.

The dependence of the resonant frequency on the perpendicularly applied magnetic field is shown for $5 \mathrm{~K}$ and $300 \mathrm{~K}$ in Fig. 9. Linearity indicates that Kittel's resonance condition also holds true for the ferrimagnetic sphere at both temperatures. The gyroscopic ratio, as measured from the slope, is given by $27.7(4) \mathrm{GHz} / \mathrm{T}$ at $300 \mathrm{~K}$ and $27.82(9) \mathrm{GHz} / \mathrm{T}$ at $5 \mathrm{~K}$, which is within the experimental error of the standard value of $28 \mathrm{GHz} / \mathrm{T}$. It is worth noting that in comparison to the polycrystalline rectangular cuboid (seen in Fig. 5) the demagnetising fields are substantially reduced. 


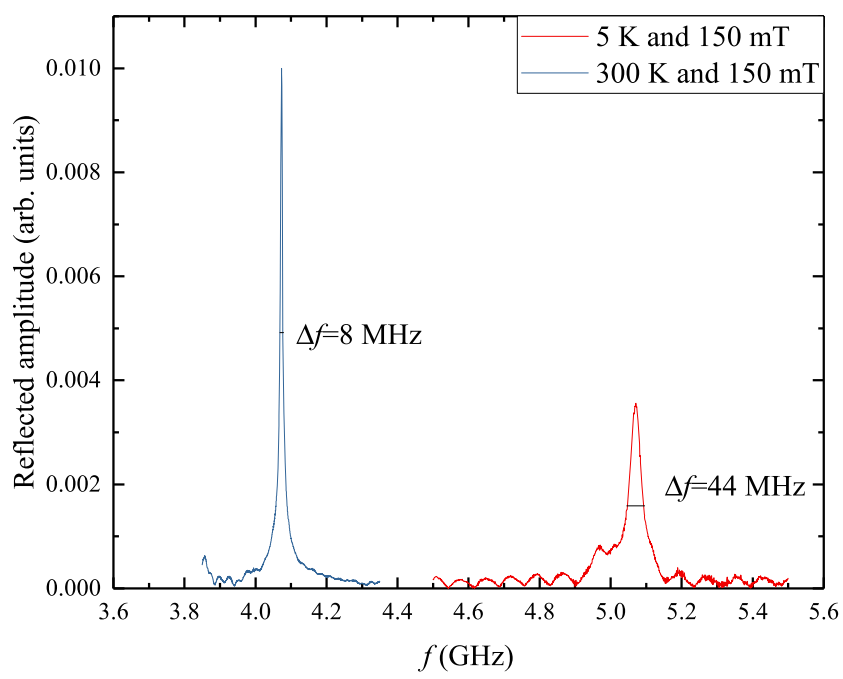

FIG. 8. VNA-FMR resonant peaks for a single crystal YIG sphere in a nominal $150 \mathrm{mT}$ applied magnetic field. Strong coupling of the electrostatic modes of the microstrip with the resonant mode leads to splitting and linewidth broadening, as observed in the $5 \mathrm{~K}$ spectrum.

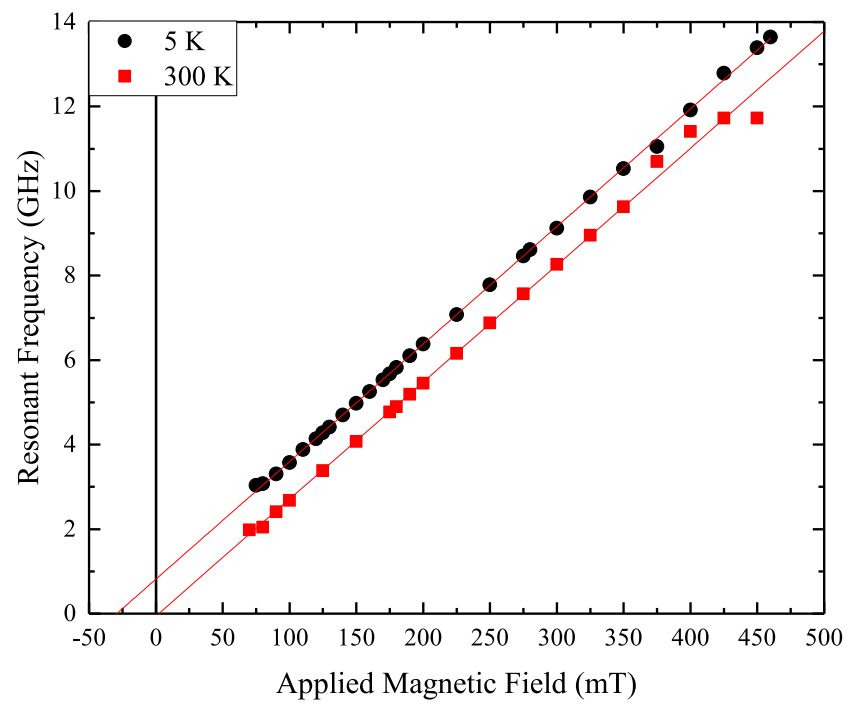

FIG. 9. Dependence of the resonant frequency on the external perpendicular field for the single crystal sphere, where demagnetising fields are reduced in comparison with the rectangular cuboid sample. The resonant frequencies are determined by recording the position of the maximum amplitude of the experimental data for each applied magnetic field.

\section{A. SQUID detection: $\Delta m_{z}$ athermal regime detection}

To reduce the $1 / f$ noise and narrowband the measured output signal, the $\mathrm{GHz}$ excitation signal provided by the $100 \mathrm{MHz}-20 \mathrm{GHz}$ signal generator is chopped. The microwave pulses (wave-trains) have 50\% duty cycle for all presented experiments. A shorter duty cycle has the effect of lowering the detected amplitude essentially linearly in the vicinity of $50 \%$. Very short duty cycles lead to unnecessary spectral broadening (as of their much wider spectral bandwidth) and are only useful to minimize the integral heat-load on the sample and the $50 \Omega$ terminator. The frequency of chopping dictates the operating regime of the measurement. At a chopping frequency of $1 \mathrm{kHz}$, resonant absorption is in the athermal regime,

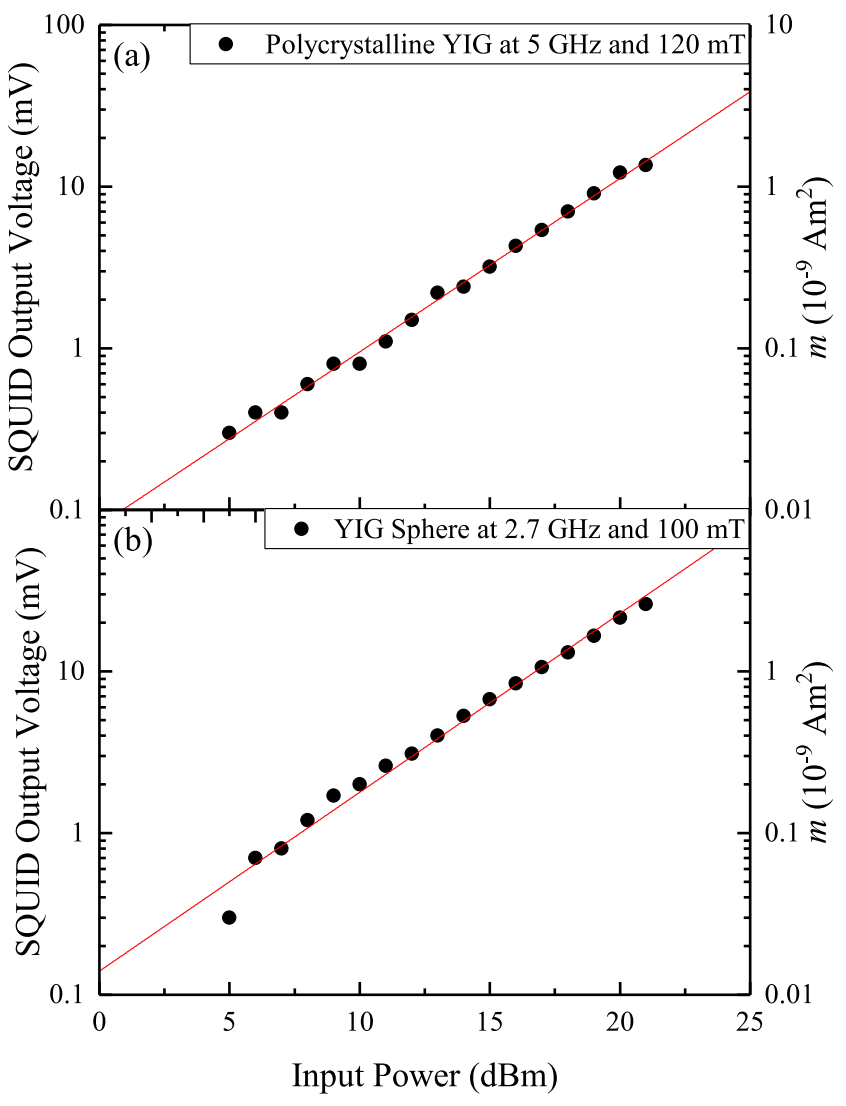

FIG. 10. Dependence of the SQUID output on the input power for (a) polycrystalline and (b) single crystal YIG is quadratic (on a linear microwave field scale) as expected from the cosine expansion at small angles. The secondary $\mathrm{y}$-axis shows the corresponding change of macrospin moment projection.

where the change in the lock-in demodulated and processed SQUID output voltage is directly proportional to the change in the $z$ projection of the macro-spin. Figure 10 shows the dependence of the SQUID output voltage on the input power for both the poly- and single-crystalline specimens, when excited at $5 \mathrm{GHz}$ in a $120 \mathrm{mT}$ field and $2.7 \mathrm{GHz}$ and $100 \mathrm{mT}$, respectively. The relation on a linear scale is shown to be quadratic, as expected from the cosine expansion at small angles.

In a static longitudinal magnetic field, the frequency is swept and resonance is indicated by a sharp increase in the SQUID output voltage. Figure 11 displays a sample SQUID resonance spectrum obtained for the polycrystal in a nominal $50 \mathrm{mT}$ applied magnetic field. The spectrum indicates a primary peak in the resonance absorption at $3.02 \mathrm{GHz}$ followed by a secondary weaker peak at $3.25 \mathrm{GHz}$. Figure 12 compares the SQUID detected resonance frequency of the primary resonant mode with the VNA obtained $f_{\text {res }}$ as a function of the applied field. Excellent agreement is shown between the two FMR detection methods and the linear high-bias field regime of Kittel dynamics. ${ }^{17}$ The gyromagnetic ratio $\gamma$ and demagnetising field are shown to be 27.4(2) GHz/T and $61.9 \mathrm{mT}$, respectively.

To compare the VNA and SQUID-detected resonance in the single-crystal sphere, two resonant peaks in a static $150 \mathrm{mT}$ field at $300 \mathrm{~K}$ are closely examined in Fig. 13. The characteristic sharp Lorentzian profile is observed, with the FWHM of the VNA-detected spectra measured as $11 \mathrm{MHz}$. The SQUID 


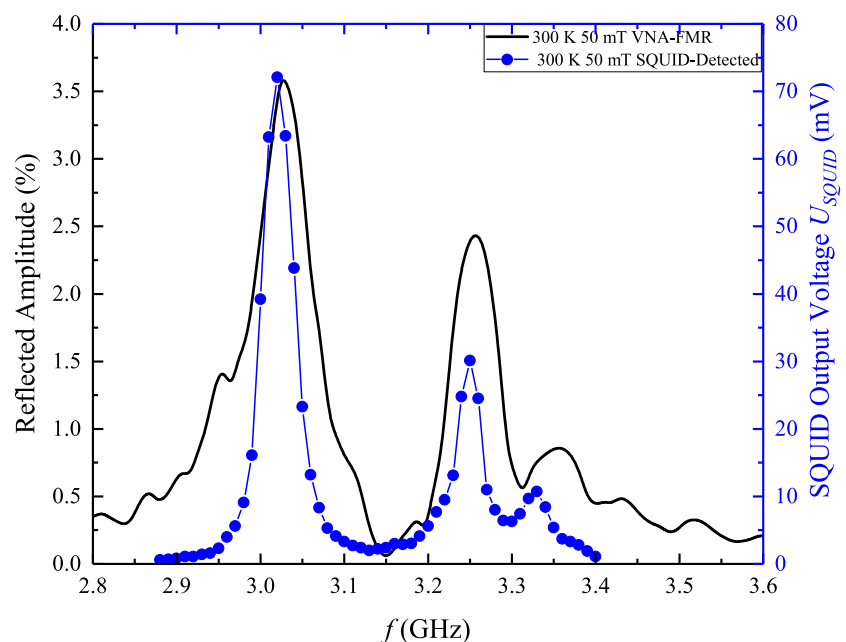

FIG. 11. Dependence of both the VNA-FMR measured reflected amplitude and, on a secondary $y$-axis, the SQUID output voltage on the applied excitation frequency in a nominal $\mu_{0} H=50 \mathrm{mT}$ field for the polycrystalline YIG.

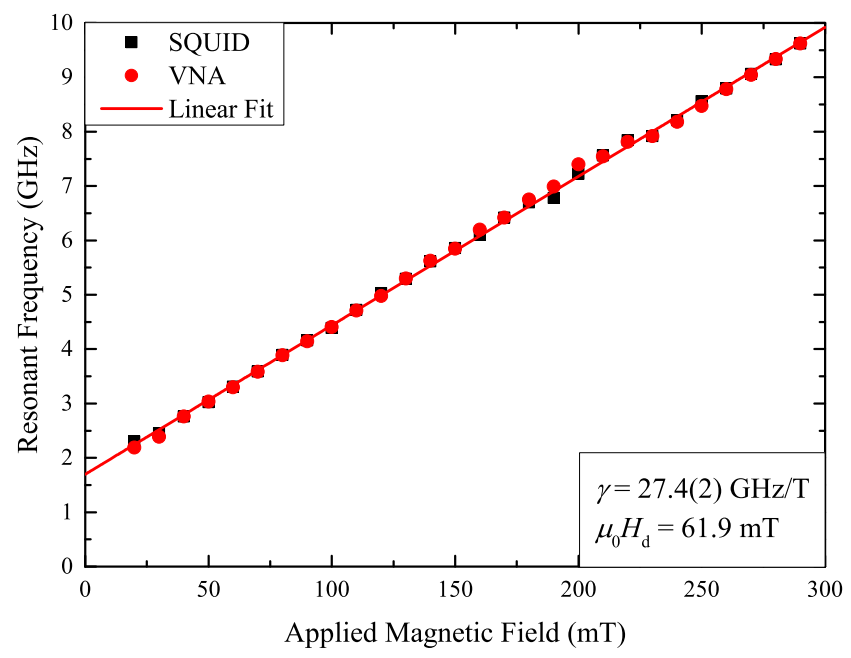

FIG. 12. Resonant frequency (GHz) as a function of applied magnetic field for both absolute SQUID and VNA-FMR detection for the polycrystalline YIG.

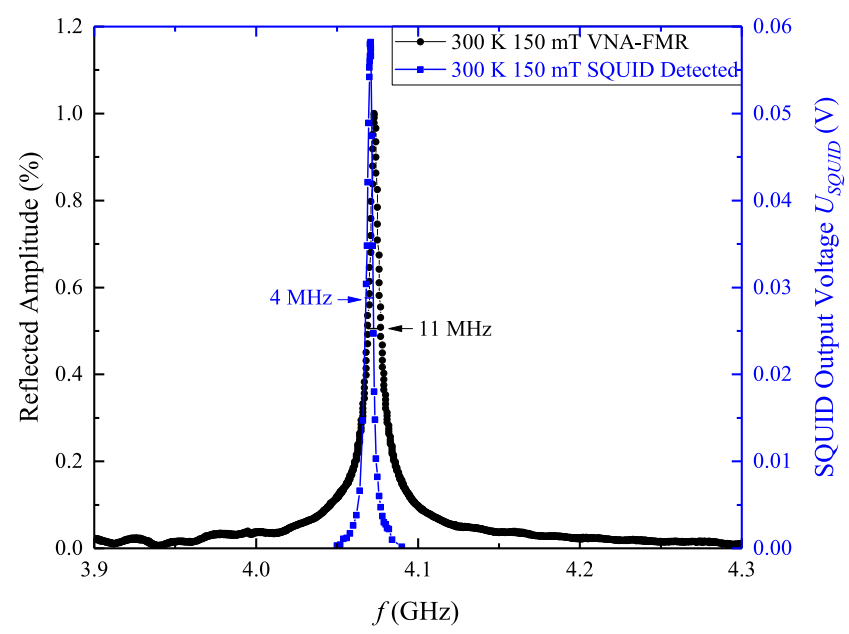

FIG. 13. Resonant peaks for the single crystal sphere detected by absolute SQUID and VNA in a static $150 \mathrm{mT}$ field are compared. For the case of the SQUID-detection, a sharp linewidth of $4 \mathrm{MHz}$ is observed.

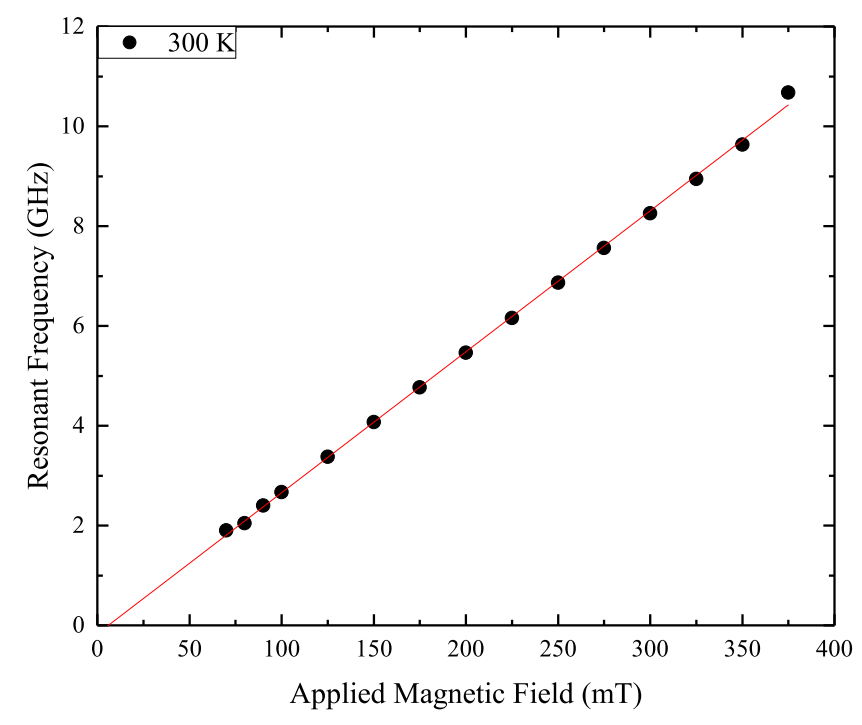

FIG. 14. Relationship between the resonant frequency and the applied field shown by athermal absolute SQUID detection for the single-crystal YIG sphere.

detected method measures a much sharper resonance linewidth of just $4 \mathrm{MHz}$. This is attributed to the intermediate frequency filtering within the VNA itself. The linewidth, in the case of SQUID-detection, is limited by the chopping or modulation frequency or, if that is sufficiently low, by the fundamental broadening for the sample. In the case of single-crystalline YIG spheres, this has been shown to be related to the quality of the surface polishing. ${ }^{25}$ Figure 14 shows the correlation between the SQUID-detected resonant frequency and the applied magnetic field, which displays good agreement with the $300 \mathrm{~K}$ VNA-FMR scan in Fig. 9. The gyromagnetic ratio is determined to be $28.2(4) \mathrm{GHz} / \mathrm{T}$.

\section{B. SQUID detection: Thermal regime detection}

At low chopping frequency $(<50 \mathrm{~Hz}), \mathrm{FM}$ resonant absorption generates oscillatory thermal waves, which induce lowering of the macro-spin's projection along the $z$-axis. Here, we take a macroscopic view of heat-wave propagation across the structure, with the typical frequencies of excited waves ranging from $\mathrm{mHz}$ to several tens of $\mathrm{kHz}$. The corresponding wavelengths would be on the order of $\mu \mathrm{m}$ to several centimetres, depending on the thermal conductance and heat capacitance of the various materials involved (sample, support structure-microstrip, varnish, etc.). These are not the shortwavelength phonons of YIG and are not explicitly quantized, but rather possess a very smooth continuous spectrum and wavelength dispersion. The sample would essentially never be uniformly heated by the precession losses. The dissipation (heat source distribution) would vary spatially, depending on the macroscopic magnetostatic modes excited (i.e., would be substantially smaller at the modes' nodes), while the heat sinking is typically also rather non-uniform, with the majority of it provided by Newtonian heat conduction to the microstrip structure and ultimately to the thermal bath. A substantially lower thermal conductivity is effected by gaseous convection (for the relatively low pressures or residual helium in the sample space, pressures are typically $10^{-2}$ mbar or lower) and by IR 
emission, which only becomes important at high temperatures (as it varies as $\approx T^{4}$ ). The dynamic thermal gradients across the sample can be substantial and at high microwave powers can reach several $\mathrm{K} / \mathrm{mm}$. In this regime, detection of $\mathrm{d} m_{z} / \mathrm{d} T$ is demonstrated. Figure 15 shows a direct comparison between resonance spectra obtained in the athermal and thermal regimes at different experimental temperatures $(4 \mathrm{~K}-400 \mathrm{~K})$ for the polycrystalline sample. In the thermal regime, the SQUID output voltage is shown to be substantially larger, especially at lower temperatures. Figure 16(a) shows the thermal conductivity $\sigma$ of bulk YIG with varying temperature, which displays a clear maximum at $20 \mathrm{~K}$. In this regime, the heat capacity $c$ shown in Fig. 16(b) has a prominent role in the resonant excitation and governs the SQUID output voltage. Values of both $\sigma$ and $c$ are obtained from a number of sources ${ }^{26-28}$ and are interpolated ( $\beta$-splined) together to form a complete picture of the thermal properties as a function of changing temperature. The thermal time constant, defined as $\tau=c \rho_{Y I G} / \sigma A$, where $\rho_{Y I G}=5.11 \times 10^{3} \mathrm{~kg} / \mathrm{m}^{3}$ and $A$ is the surface area of the rectangular prism of YIG, is dominated by the heat capacity and tends to zero at low temperatures as is shown in Fig. 17. If the thermal coupling of the sample to the SQUID heat bath is

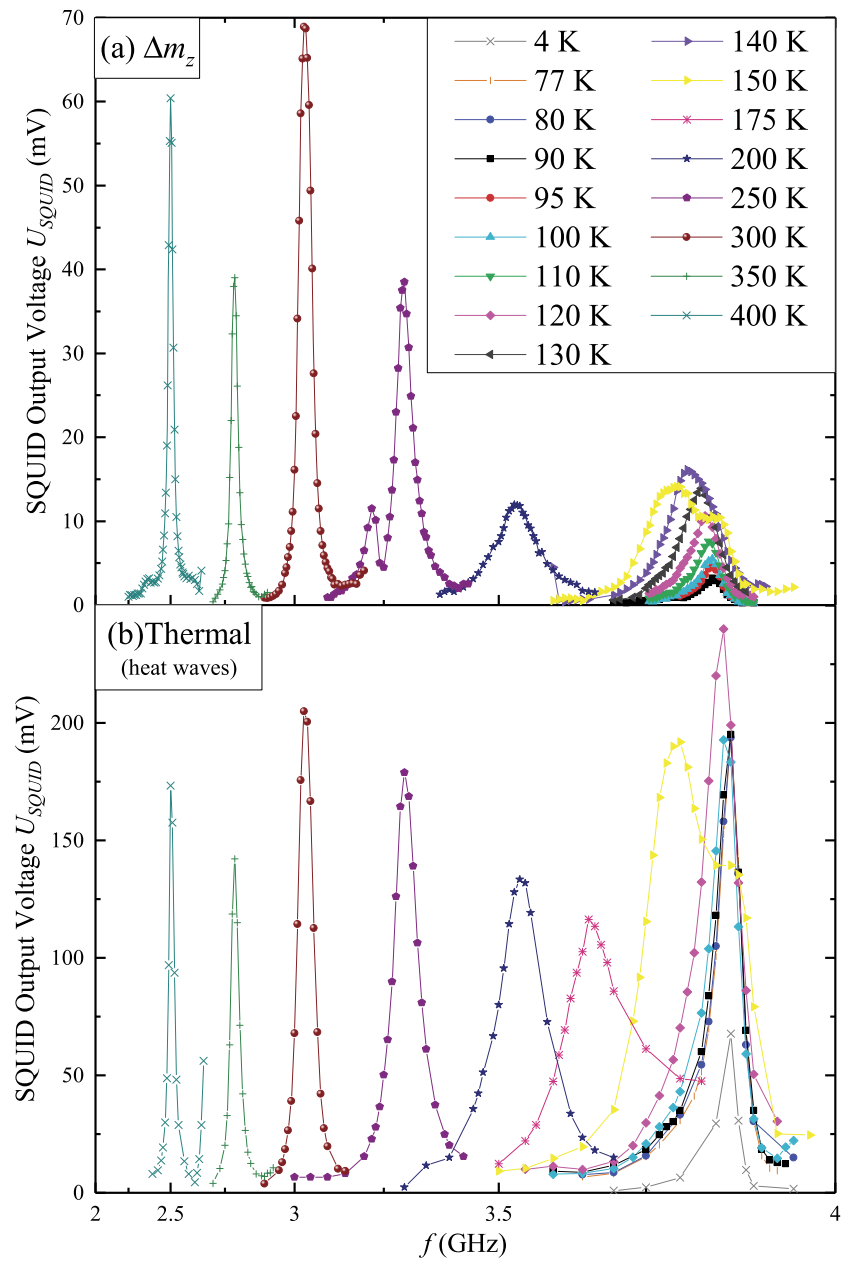

FIG. 15. Resonance spectra obtained at different temperatures ( $90-400 \mathrm{~K}$ ) by (a) monitoring the SQUID output voltage which is proportional to the change in the projection of the magnetisation along the applied-field direction. (b) In this regime, the SQUID output voltage is proportional to the change in $m_{z}$ resulting from a temperature change $\mathrm{d} T$ caused by resonant heating.

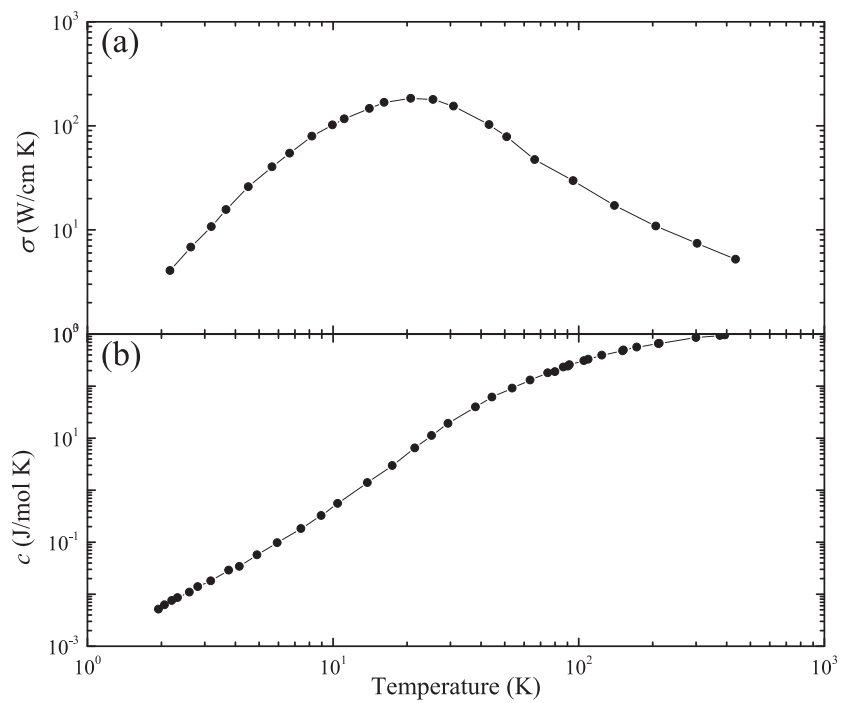

FIG. 16. Thermal properties of bulk YIG with changing temperature. (a) The thermal conductivity shows a clear maximum around $20 \mathrm{~K}$. (b) The heat capacity governs the SQUID output voltage.

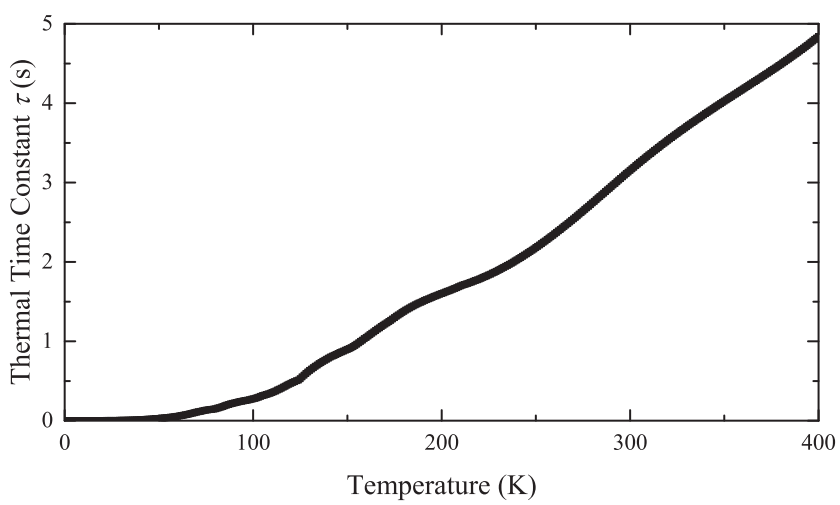

FIG. 17. The thermal time constant is governed by the heat capacity and constrains to zero at low temperatures.

considered as an equivalent RLC circuit, as in Fig. 18(b), the thermal impedance of the system can be effectively simulated. Accounting for this impedance and the fit to the magnetisation curve seen in the inset of Fig. 19, the material dependent $\mathrm{d} m_{z} / \mathrm{d} T$ can be modeled, as seen in Fig. 18(a). Figure 19 shows

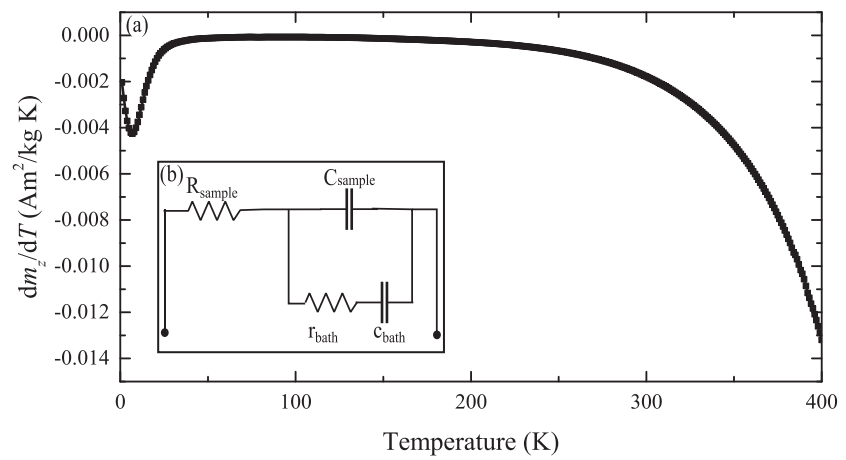

FIG. 18. (a) Simulated $\mathrm{d} m_{z} / \mathrm{d} T$. (b) Inset shows a rough schematic of the equivalent circuit model, illustrating the thermal coupling of the sample to the SQUID heat bath. 


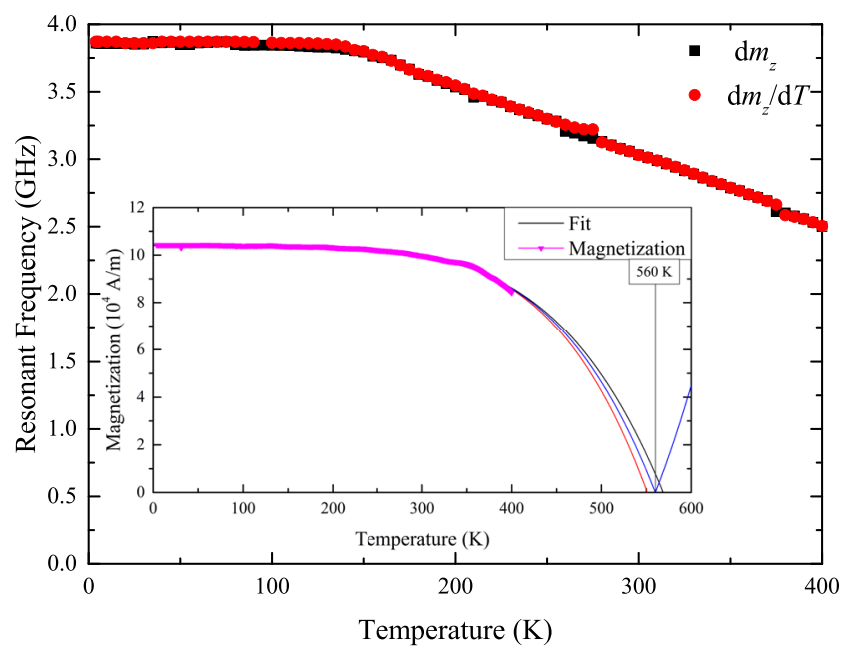

FIG. 19. Temperature dependence of the resonant frequency detected by athermal $\left(\mathrm{d} m_{z}\right)$ and thermal $\left(\mathrm{d} m_{z} / \mathrm{d} T\right)$ regime measurements of the SQUID output voltage, for bulk YIG in a $50 \mathrm{mT}$ static field. Inset shows the temperature dependence of the magnetisation for the polycrystalline YIG.

the variation of resonance frequency with temperature in a static $50 \mathrm{mT}$ magnetic field. Both regimes show the resonance frequency plateauing below a critical temperature $(T=160 \mathrm{~K})$. The phase shift between the lock-in demodulated voltage and the "chopped" input signal at low temperatures, shown in Fig. 20, suggests a transition between the athermal and thermal excitation regimes. The behaviour of the thermal system is essentially one of a conventional low-pass filter; the theoretical limit for the phase shift is $-90^{\circ}$. In the limit of long thermal time constants, on the order of seconds to several seconds, which is the case for weakly coupled samples of millimetric dimensions, close to room temperature, the thermal time constant can be measured by the conventional pulsed method, i.e., by abruptly switching on and off the microwave excitation and tracking the heat up and cool down exponents. Such an approach is illustrated by the raw SQUID signals visualized on the inset of Fig. 23. The same approach becomes impractical for thermal time constants shorter than $100 \mathrm{~ms}$, at which

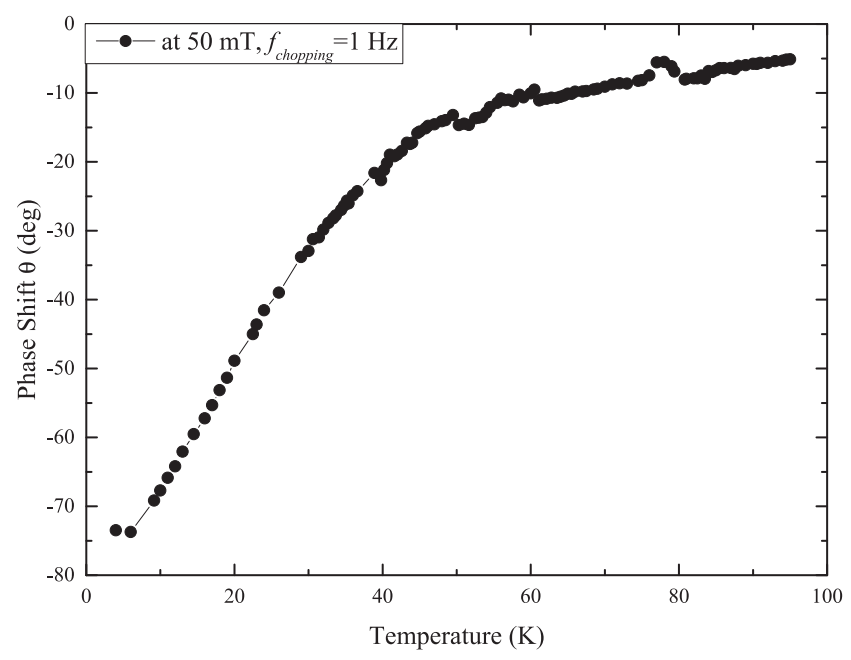

FIG. 20. Monitoring of the phase shift in a static $50 \mathrm{mT}$ field and $1 \mathrm{~Hz} f_{\text {chopping }}$ at low temperatures suggests a transition between the athermal and thermal regimes. point an AC de-phasing approach becomes more practical. It is, in principle, possible to mount millimetre and sub-millimetre samples on a thin wire ( $25 \mu \mathrm{m}$ diameter, gold-plated tungsten) symmetric line (Twinax ${ }^{\circledR}$ ) assembly, in order to perform absolute calorimetry. Such additional functionality goes beyond the scope of this manuscript.

\section{SQUID detection: Thermal regime continuous wave resonant absorption}

As displayed in Fig. 21, continuous wave (CW) excitation with thermal detection is achievable. Absorption spectra are obtained by the direct digitization of the output voltage as the sample is subjected to a continuous swept frequency excitation. This, of course, results in a quasi-static non-uniform temperature distribution (thermal gradient) across the sample and underlying structure, which varies in time on the scale of the duration of the frequency span (of the order of minutes). Figure 22 shows a colour map of the resonant modes at varying temperatures. In agreement with Fig. 6, two primary absorption modes, converging at very low and high temperatures, are displayed. Once again we see strong coupling of the FM resonant modes and electrostatic modes of the microstrip transmission line at specific frequencies.

Figure 23 shows a typical thermal SQUID-FMR absorption curve for the single-crystal YIG sphere at $25 \mathrm{~K}$, again obtained by direct oscilloscope digitization of the SQUID voltage.

The level of thermal coupling between the sample and the cryostat's isothermal shield ultimately determines the rate at which the excitation frequency can be swept. At very low sweep rates, comparable with the characteristic time-constant of the proportional integral derivative (PID) temperature controller, the back action of the same leads to line shape distortion and significant attenuation of the amplitude of the detected signal. Moreover, the conventional strategy of using different optimised PID values at different temperatures makes it much more tractable to analyze the behaviour of small samples of low heat capacity or when $\tau_{\text {sample }}<<\tau_{\text {cryostat }}$. As this version

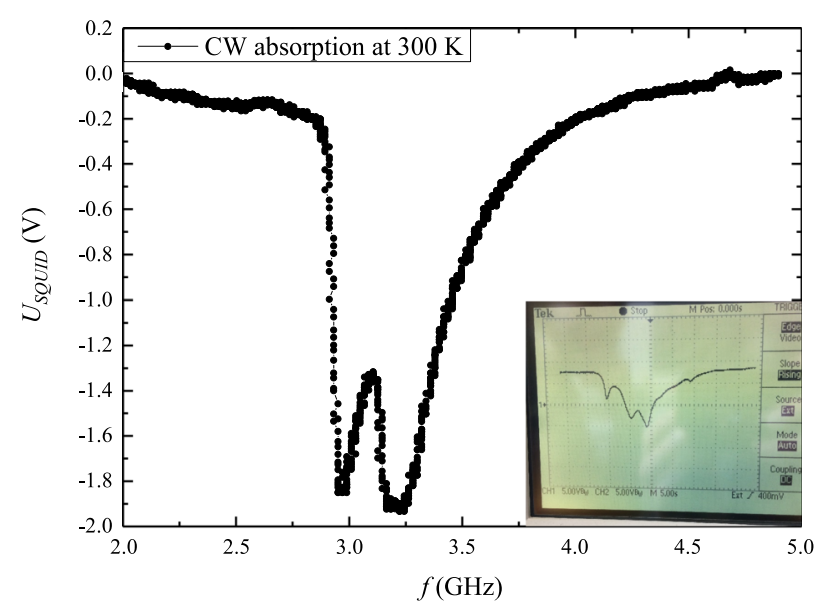

FIG. 21. CW Microwave excitation and absorption detection via direct digi-

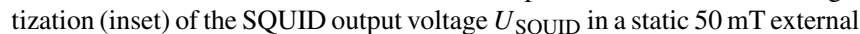
field. Two resonant modes corresponding to $x$ - and $y$-axis dipole excitations are observed. The $z$-axis mode is not sufficiently excited in this geometry. 


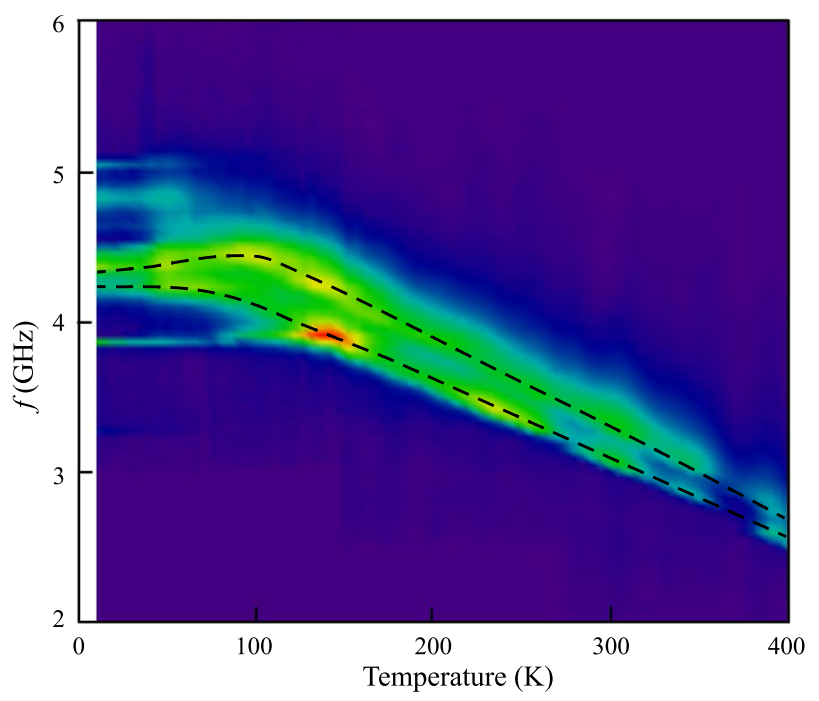

FIG. 22. Continuous wave microwave absorption indicates two resonant modes for the polycrystalline cuboid which converge at high and low temperatures. This finding is congruent with the VNA-FMR map displayed in Fig. 6.

of the technique does not require amplitude (chopping) or frequency modulation, the ultimate frequency resolution can be much higher and even allow for the measurement of paramagnetic resonance.

The temperature dependence of the thermally detected resonant frequency for the YIG sphere is shown in Fig. 24. The signal is again strongest at low temperature, where the thermal time constant tends to zero and resonant signals are measurable at temperatures down to $5 \mathrm{~K}$, limited by the power dissipation at the $50 \Omega$ termination resistor and the amount of available cooling power. Above $100 \mathrm{~K}$, as $\tau$ becomes significant (see Fig. 17), linewidth broadening becomes especially pronounced. The inhomogeneity of the RF excitation excites

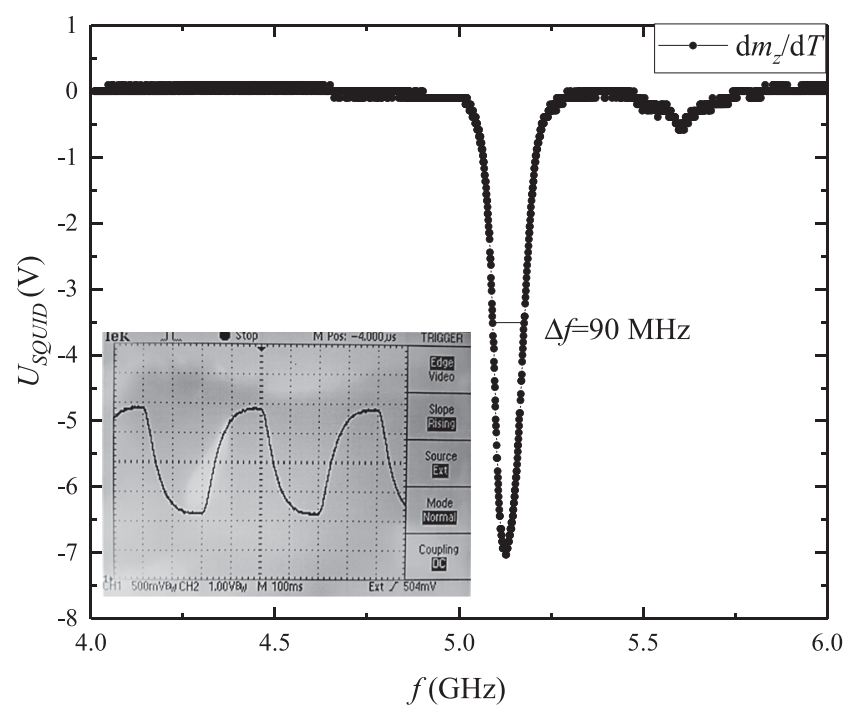

FIG. 23. CW Microwave excitation and absorption detection via direct digitization of the SQUID output voltage $U_{\text {SQUID }}$ at $20 \mathrm{~K}$. For the case of the single crystal YIG sphere, a single sharp resonant peak is observed. The inset shows the time variation of the SQUID output voltage for a slow modulation (chopping) of the microwave power-the period is $400 \mathrm{~ms}$.

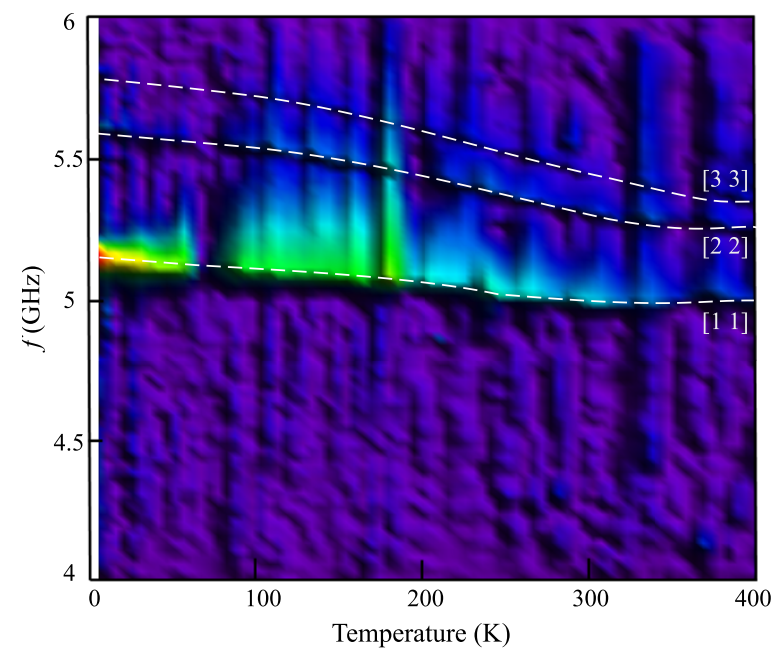

FIG. 24. The shift in the resonant frequency with changing temperature for the single-crystal sphere is less pronounced than that observed for the bulk polycrystalline piece as shown in Fig. 22. In addition to the primary [1 1$]$ mode, two magnetostatic modes are identified [2 2] and [3 3]. It could be noted that a much weaker mode is seen at higher frequencies, which may be identified as the [2 1] mode.

higher-order non-uniform resonant modes, the strongest of which are indexed as [1 1], [2 2], and [3 3]..$^{13,29,30}$ The shift in the resonant frequency of the [1 1] mode with increasing temperature is shown to be much less dispersive than that observed for the polycrystal (Fig. 22) and also less dispersive than the higher modes. This is likely due to the higher contribution of surface anisotropy to the resonance conditions for the higher modes. As anisotropy tends to gap the dispersion relations of the magnetostatic modes, their population intensities tend to drop correspondingly, at low temperatures.

The FR4 actually provides some advantages in the thermal detection regime. At low temperatures, the additional change in moment and susceptibility of the paramagnetic impurities in the board of the microstrip (which itself can be thermally well anchored to the sample) actually improves sensitivity. This is due to the hyperbolic divergence of the susceptibility of these impurities, at low temperature. A small change in temperature of the region of the microstrip (in vicinity of the sample) due to the sample's resonant absorption produces a large change in the overall magnetic moment and the corresponding flux coupled to the SQUID gradiometer. This strongly suggests that an alternative approach to sensitivity improvement would be to immerse the sample in a "cocoon" of an "ideal" paramagnetic salt such as Mohr's salt or ammonium iron (III) sulphate dodecahydrate (ferric ammonium alum-FAA). Of course, such an approach is only really beneficial at temperatures below a few $\mathrm{K}$, for an applied field of $\mu_{0} H \sim \mathrm{T}$.

\section{SUMMARY}

In summary, the design and operation of a contactless SQUID-based FMR detection system, capable of operating in a broad range of experimental temperatures, is reported. SQUID-FMR detection has higher absolute sensitivity than conventional cavity or stripline methods with the added advantage of gaining a qualitative insight into the precession 


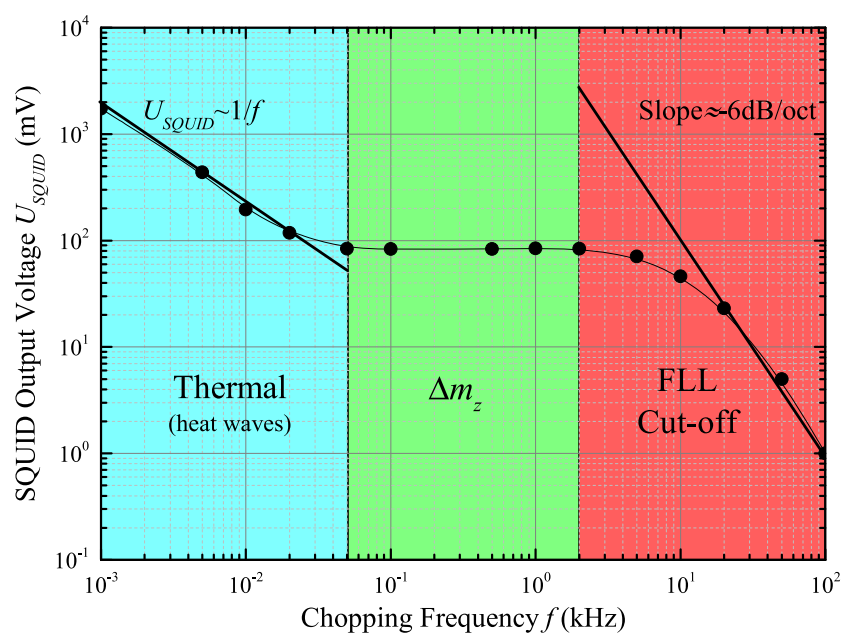

FIG. 25. SQUID output voltage as a function of the chopping frequency of the $\mathrm{GHz}$ excitation for the polycrystalline cuboid. Three regimes are displayed: thermal $(1-20 \mathrm{~Hz})$ where resonant heating changes the projection of $m_{z}$, athermal $\Delta m_{z}(50 \mathrm{~Hz}-2 \mathrm{kHz})$ where the change in the projection during resonant absorption is directly measured, and the flux-locked loop cutoff.

dynamics. In addition to SQUID-detection, conventional VNA-FMR detection is possible and the two methods are complimentary. This module has been constructed to fit the sample space of a Quantum Design magnetic property measurement system MPMS XL ${ }^{10}$ but is compatible with most commercial SQUID magnetometer systems. By chopping the external microwave excitation at different frequencies, the system may be operated in two regimes, thermal and athermal, which give absolute values of $\Delta m_{z}$ and $\mathrm{d} m_{z} / \mathrm{d} T$, respectively, as the material is driven at its resonance frequency. Figure 25 shows the SQUID output voltage as a function of the chopping frequency applied to the $\mathrm{GHz}$ excitation for the polycrystalline cuboid. At low frequency $(<50 \mathrm{~Hz})$, resonant absorption generates oscillatory thermal waves which alter the projection of $m_{z}$. Between $50 \mathrm{~Hz}$ and $2 \mathrm{kHz}$ measurement is stable and allows for direct detection of $\Delta m_{z}$. Above $100 \mathrm{kHz}$, the slew-rate of the SQUID-system is exceeded and the SQUID no longer remains flux-locked. For each material and sample geometry, the cutoff chopping frequency for each regime will shift. Figure 26 defines the operation regimes for the single-crystal sphere. The transition between the thermal and athermal regimes of detection is shifted towards lower frequencies in this case, on account of the worse thermal coupling between the sample and the microstrip carrier which is limited by the geometrical area of contact provided by the mounting varnish.

The operation of the SQUID-FMR module has been successfully used to observe the magnetisation dynamics of two resonant modes in a rectangular piece of bulk $\mathrm{Y}_{3} \mathrm{Fe}_{5} \mathrm{O}_{12}$ with varying magnetic field and temperature. Potentially, all three detection strategies demonstrated could be combined (in realtime) with DC and AC magnetisation measurements, dramatically expanding the amount of sample characterisation data and allowing for the quantitative discrimination between dissipative and dispersive resonant and non-resonant effects. The instrumentation is presently limited to the characterisation of bulk materials with low damping in the microwave region. In order to gain the additional ability to characterise thin films,

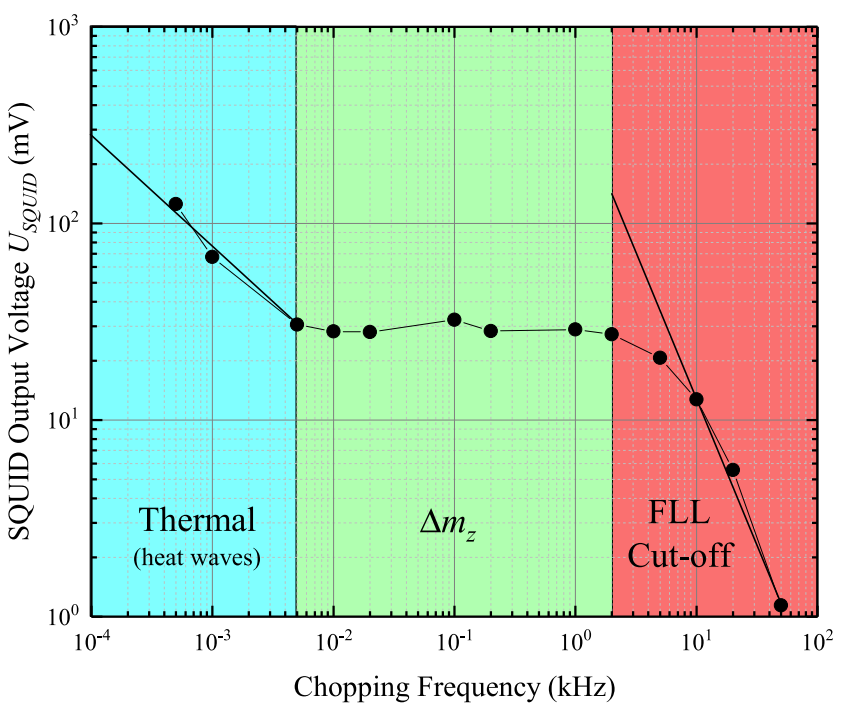

FIG. 26. SQUID voltage as a function of the chopping frequency of the $\mathrm{GHz}$ excitation for the single crystal YIG sphere. Again, three regimes are displayed: thermal $(0.1-2 \mathrm{~Hz})$, athermal $\Delta m_{z}(5 \mathrm{~Hz}-2 \mathrm{kHz})$, and the flux-locked loop cutoff.

random/templated nano-structures, or metallic systems, the power of the excitation would need to be increased, but it is currently limited by the fact that the signal generator is operated in a single-port configuration and the power is dissipated at the $50 \Omega$ termination at the end of the microstrip. As such, increasing the power would lead to an increase in the residual heating within the sample space, which may be detrimental during low temperature measurements. A second transmission line can, in-principle, be used to enable the dissipation of much higher microwave power externally to the sample environment. This would enable the measurement of ferromagnetic resonance at very high excitation power (in excess of tens or even hundreds of watts) and access to very non-linear dynamics, at large precession angles. The same is important, for example, for the definition of the fundamental limits of intermodulation distortion in critical high-performance microwave devices, such as filters and stripline circulators. In addition to this, magnetically clean dielectric materials (alumina, quartz, etc.) will be investigated as alternatives to the FR4 for absolute FMR detection.

The strategies discussed can be generalised to vacuum cavity waveguides in order to access higher frequencies $(>20 \mathrm{GHz})$ that are otherwise inaccessible. For frequencies in the range of $20 \mathrm{GHz}-50 \mathrm{GHz}$, this can be done in sets of shorted discrete frequency cavities and beyond $50 \mathrm{GHz}$ it would even be possible to use a return track geometry. Provided the power can be delivered to and absorbed by the sample, FMR, EPR, and high-field-NMR can all be detected using SQUID-based techniques.

\section{ACKNOWLEDGMENTS}

This publication has emanated from research conducted with the financial support of Science Foundation Ireland (SFI) under Grant No. SFI/12/RC/2278 and the European Commission under the TRANSPIRE FET Open project EC 737038. The authors would like to acknowledge fruitful 
discussions with R. Hapanowicz, D. Polancic and R. Dumas from Quantum Design Inc. The authors wish to thank S. Bulja and P. Rulikowski from Alcatel-Lucent Inc. for providing some of the polycrystalline YIG samples used.

${ }^{1}$ J. H. E. Griffiths, Nature 158, 670 (1946).

${ }^{2}$ W. Yager and R. Bozorth, Phys. Rev. 72, 80 (1947).

${ }^{3}$ J. A. Sidles, J. L. Garbini, K. J. Bruland, D. Rugar, O. Züger, S. Hoen, and C. S. Yannoni, Rev. Mod. Phys. 67, 249 (1995).

${ }^{4}$ M. M. Midzor, P. E. Wigen, D. Pelekhov, W. Chen, P. C. Hammel, and M. L. Roukes, J. Appl. Phys. 87, 6493 (2000).

${ }^{5}$ G. Boero, S. Rusponi, P. Bencok, R. S. Popovic, H. Brune, and P. Gambardella, Appl. Phys. Lett. 87, 1 (2005).

${ }^{6}$ R. Meckenstock, M. Moller, and D. Spoddig, Appl. Phys. Lett. 86, 112506 (2005).

${ }^{7}$ M. Moller, D. Spoddig, and R. Meckenstock, J. Appl. Phys. 99, 08J310 (2006).

${ }^{8}$ T. Gerrits, P. Krivosik, M. L. Schneider, C. E. Patton, and T. J. Silva, Phys. Rev. Lett. 98, 207602 (2007).

${ }^{9}$ M. Wurm, J.-P. Brison, and J. Flouquet, Rev. Sci. Instrum. 69, 1456 (1998).

${ }^{10}$ Quantum Design Magnetic Property Measurement System MPMS® XL, Quantum Design, 6325 Lusk Boulevard, San Diego, CA 92121-3733, USA.

${ }^{11}$ R. Douglass, Phys. Rev. 129, 1132 (1963).

${ }^{12}$ T. An, V. I. Vasyuchka, K. Uchida, A. V. Chumak, K. Yamaguchi, K. Harii, J. Ohe, M. B. Jungfleisch, Y. Kajiwara, H. Adachi, B. Hillebrands, S. Maekawa, and E. Saitoh, Nat. Mater. 12, 549 (2013).
${ }^{13}$ N. J. Lambert, J. A. Haigh, and A. J. Ferguson, J. Appl. Phys. 117, 41 (2015). ${ }^{14}$ N. Vlietstra, B. J. Van Wees, and F. K. Dejene, Phys. Rev. B 94, 035407 (2016); e-print arXiv:1601.05605.

${ }^{15}$ M. Haertinger, C. H. Back, J. Lotze, M. Weiler, S. Geprägs, H. Huebl, S. T. B. Goennenwein, and G. Woltersdorf, Phys. Rev. B 92, 054437 (2015).

${ }^{16}$ A. J. Baden Fuller, Ferrites at Microwave Frequencies (Peter Peregrinus, 1987), pp. 12-14.

${ }^{17}$ C. Kittel, Phys. Rev. 73, 155 (1948).

${ }^{18}$ I. V. Kolokolov, V. S. L'vov, and V. B. Cherepanov, Sov. Phys. JETP 59, 1131 (1984), available at http://www.jetp.ac.ru/cgi-bin/e/index/e/59/5/ p1131? a $=$ list

${ }^{19}$ A. B. Harris, Phys. Rev. 132, 2398 (1963).

${ }^{20}$ E. A. Turov, M. I. Kurkin, V. V. Men'shenin, and V. V. Nikolaev, Phys. Met. Metallogr. 103, 446 (2007).

${ }^{21}$ V. Cherepanov, I. Kolokolov, and V. L'vov, Phys. Rep. 229, 81 (1993).

${ }^{22}$ M. Sparks, J. Appl. Phys. 36, 1570 (1965).

${ }^{23}$ J. F. Dillon, Phys. Rev. 105, 759 (1957).

${ }^{24}$ J. F. Dillon, Phys. Rev. 111, 1476 (1958).

${ }^{25}$ R. C. LeCraw, E. G. Spencer, and C. S. Porter, Phys. Rev. 110, 1311 (1958).

${ }^{26}$ G. A. Slack and D. W. Oliver, Phys. Rev. B 4, 592 (1971).

${ }^{27}$ M. Guillot, F. Tchéou, A. Marchand, P. Feldmann, and R. Lagnier, Z. Phys. B Condens. Matter 44, 53 (1981).

${ }^{28}$ S. M. Rezende, R. L. Rodriguez-Suarez, J. C. Lopez Ortiz, and A. Azevedo, Phys. Rev. B 89, 134406 (2014).

${ }^{29}$ P. C. Fletcher and R. O. Bell, J. Appl. Phys. 30, 687 (1959).

${ }^{30}$ L. R. Walker, Phys. Rev. 105, 390 (1957). 